\title{
Constraining Right Handed Gauge Boson Mass from Lepton Number Violating Meson Decays in a Low Scale Left Right Model
}

\author{
Sanjoy Mandal*i \\ The Institute of Mathematical Sciences, C.I.T Campus, Taramani, Chennai 600 113, India \\ E-mail: smandal@imsc.res.in
}

\begin{abstract}
We analyze the lepton number violating (LNV) meson decays that arise in a TeV scale Left Right Symmetry model. The right handed Majorana neutrino $N$ along with the right handed or Standard Model gauge bosons mediate the meson decays and provide a resonant enhancement of the rates if the mass of $N\left(M_{N}\right)$ lies in the range $\sim(100 \mathrm{MeV}-5 \mathrm{GeV})$. Using the expected upper limits on the number of events for the LNV decay modes $M_{1}^{+} \rightarrow \ell^{+} \ell^{+} \pi^{-}\left(M_{1}=B, D, D_{s}, K\right)$, we derive constraints plausible on the mass of the right handed charged gauge boson by future searches at the ongoing NA62 and LHCb experiments at CERN, the upcoming Belle II at SuperKEK, as well as at the proposed future experiments, SHiP and FCC-ee. These bounds are complimentary to the limits from same-sign dilepton search at Large Hadron Collider (LHC). The very high intensity of Charmed mesons expected to be produced at SHiP will result in a far more stringent bound, $M_{W_{R}}>18.4 \mathrm{TeV}$ (corresponding to $M_{N}=1.46 \mathrm{GeV}$ ), than the other existing bounds from collider and neutrinoless double beta decay searches.
\end{abstract}

The 39th International Conference on High Energy Physics (ICHEP 2018)

4-11 July 2018

Seoul, Korea

\footnotetext{
* Speaker.

${ }^{\dagger}$ In collaboration with Manimala Mitra and Nita Sinha.
} 


\section{Introduction}

One of the most attractive framework to explain the small light neutrino masses is the Minimal Left-Right Symmetry Model (MLRSM) [1]. The light neutrino masses in this model are generated from dimension-five lepton number violating (LNV) operator that violates lepton number by two units and hence their Majorana nature can be confirmed by observing various LNV signal in experiments, such as neutrinoless double beta decay. LNV signature can also be tested through indirect searches from meson decays, $M_{1}^{+} \rightarrow \ell^{+} \ell^{+} \pi^{-}$. Right handed Majorana neutrinos with mass in the hundreds of $\mathrm{MeV}$-few $\mathrm{GeV}$ range, can be produced as an intermediate on mass shell state, resulting in a resonance enhancement of the LNV meson decay rates. The detailed study of these is the main objective of this paper [2].

In addition to the particle content of the Standard Model (SM), the model contains three right handed Majorana neutrinos $N_{R}$, and the additional gauge bosons $W_{R}$ and $Z^{\prime}$. The charged current Lagrangian for the quarks and leptons in this model have the following forms:

$$
\begin{aligned}
& \mathscr{L}_{C C}^{q}=\frac{g}{\sqrt{2}} \sum_{i, j} \bar{u}_{i} V_{i j}^{\mathrm{CKM}} W_{L \mu}^{+} \gamma^{\mu} P_{L} d_{j}+\frac{g}{\sqrt{2}} \sum_{i, j} \bar{u}_{i} V_{i j}^{\mathrm{R}-\mathrm{CKM}^{\prime}} W_{R \mu}^{+} \gamma^{\mu} P_{R} d_{j}+\text { H.c. }, \\
& \mathscr{L}_{C C}^{\ell}=\frac{g}{\sqrt{2}} \sum_{i, j} \bar{\ell}_{L_{i}} W_{L \mu}^{-} \gamma^{\mu} P_{L}\left(U_{i j} v_{L_{j}}+S_{i j} N_{j}^{c}\right)+\frac{g}{\sqrt{2}} \sum_{i, j} \bar{\ell}_{R_{i}} W_{R \mu}^{-} \gamma^{\mu} P_{R}\left(V_{i j}^{*} N_{j}+T_{i j}^{*} v_{L}^{c}\right)+\text { H.c. },
\end{aligned}
$$

where $U=\left(1-\frac{\theta^{2}}{2}\right) U_{\mathrm{PMNS}}, V=\left(1-\frac{\theta^{2}}{2}\right) V_{R}, S=\theta V_{R}, T=-\theta U_{\mathrm{PMNS}}, \theta^{2}=\frac{m_{V}}{M_{N}}$ and we have considered $V_{R}=I$.

\section{Imprint of Majorana Signature in Meson Decays}

Together with the gauge bosons $W_{R}$, or even with $W_{L}, N_{i}$ can mediate the lepton number violating meson decays, $M_{1}^{+}(p) \rightarrow \ell_{1}^{+}\left(k_{1}\right) \ell_{2}^{+}\left(k_{2}\right) M_{2}^{-}\left(k_{3}\right)$, where $M_{1}$ is a pseudoscalar, while $M_{2}$ can be a pseudoscalar or a vector meson. We assume that there are three RH neutrinos with masses in the $100 \mathrm{MeV}-5 \mathrm{GeV}$ range, that contribute in these meson decays. The Feynman diagrams for these decays are shown in Fig 1. We can write the amplitude for this pseudoscalar meson decay as,

$$
\mathscr{M}_{h_{1} h_{2}}^{P}=\mathscr{M}_{1 h_{1} h_{2}}^{P}+\mathscr{M}_{2 h_{1} h_{2}}^{P}
$$

where, $h_{1} h_{2}$ can be of different chiralities $L L, R R, L R, R L$ and the second term is obtained by interchanging the momenta $k_{1}$ with $k_{2}$ of the 2 leptons, as well as interchanging the leptonic mixing elements.

$$
\begin{gathered}
\mathscr{M}_{1}^{P}=\mathscr{M}_{1 L L}^{P}+\mathscr{M}_{1 R R}^{P}+\mathscr{M}_{1 L R}^{P}+\mathscr{M}_{1 R L}^{P} \\
=A \sum_{i}\left(M_{N_{i}}\left(S_{\ell_{1} N_{i}}^{*} S_{\ell_{2} N_{i}}^{*}\right) \frac{\bar{u}\left(k_{2}\right) k_{3} \not p\left(1-\gamma_{5}\right) v\left(k_{1}\right)}{\left(p-k_{1}\right)^{2}-M_{N_{i}}^{2}+i M_{N_{i}} \Gamma_{N_{i}}}+M_{N_{i}}\left(\frac{M_{W_{L}}^{4}}{M_{W_{R}}^{4}}\right)\left(V_{\ell_{1} N_{i}} V_{\ell_{2} N_{i}}\right) \frac{\bar{u}\left(k_{2}\right) k_{3} \not p\left(1+\gamma_{5}\right) v\left(k_{1}\right)}{\left(p-k_{1}\right)^{2}-M_{N_{i}}^{2}+i M_{N_{i}} \Gamma_{N_{i}}}\right. \\
\left.+\left(\frac{M_{W_{L}}^{2}}{M_{W_{R}}^{2}}\right)\left(S_{\ell_{1} N_{i}}^{*} V_{\ell_{2} N_{i}}\right) \frac{\bar{u}\left(k_{2}\right) k_{3}\left(\not p-k_{1}\right) \not p\left(1-\gamma_{5}\right) v\left(k_{1}\right)}{\left(p-k_{1}\right)^{2}-M_{N_{i}}^{2}+i M_{N_{i}} \Gamma_{N_{i}}}+\left(\frac{M_{W_{L}}^{2}}{M_{W_{R}}^{2}}\right)\left(V_{\ell_{1} N_{i}} S_{\ell_{2} N_{i}}^{*}\right) \frac{\bar{u}\left(k_{2}\right) k_{3}\left(\not p-k_{1}\right) \not p\left(1+\gamma_{5}\right) v\left(k_{1}\right)}{\left(p-k_{1}\right)^{2}-M_{N_{i}}^{2}+i M_{N_{i}} \Gamma_{N_{i}}}\right)(2.1)
\end{gathered}
$$

where, $A=G_{F}^{2} V_{M_{1}}^{C K M} V_{M_{2}}^{C K M} f_{M_{1}} f_{M_{2}} . M_{N_{i}}, \Gamma_{N_{i}}$ are the mass and decay width of the heavy neutrino $N_{i}$. The total decay width of heavy majorana neutrino $N_{i}$ for the considered mass range $(0.1-5 \mathrm{GeV})$ 

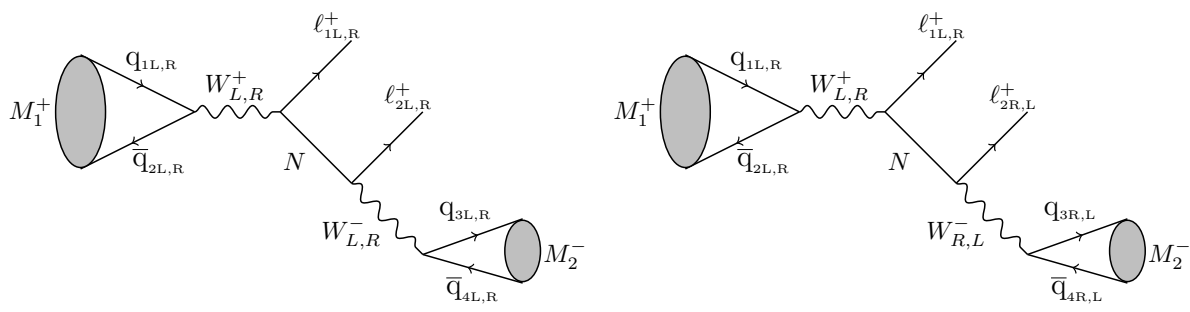

Figure 1: The Feynman diagrams for the lepton number violating meson decays. These processes produce resonance enhancement. See text for details.

is given by,

$$
\Gamma_{N_{i}}=\sum_{\ell, P} 2 \Gamma^{\ell P}+\sum_{\ell, P} \Gamma^{v_{\ell} P}+\sum_{\ell, V} 2 \Gamma^{\ell V}+\sum_{\ell, V} \Gamma^{v_{\ell} V}+\sum_{\ell_{1}, \ell_{2}\left(\ell_{1} \neq \ell_{2}\right)} 2 \Gamma^{\ell_{1} \ell_{2} v_{\ell_{2}}}+\sum_{\ell_{1}, \ell_{2}} \Gamma^{v_{\ell_{1}} \ell_{2} \ell_{2}}+\sum_{v_{\ell_{1}}} \Gamma^{\nu_{\ell_{1}} v \bar{v}} \cdot(2.2)
$$

where, $\ell=e, \mu, \tau, P^{+}=\pi^{+}, K^{+}, D^{+}, D_{s}^{+}, P^{0}=\pi^{0}, \eta, \eta^{\prime}, \eta_{c}, V^{+}=\rho^{+}, K^{*+}, D^{*+}, D_{s}^{*+}, V^{0}=\rho^{0}, \omega, \phi, J / \psi$, $\ell_{1}, \ell_{2}=e, \mu, \tau, \ell_{1} \neq \ell_{2}$. In Fig.2, we have shown the $\Gamma_{N}$ as a function of mass $M_{N}$ for different $W_{R}$ masses.

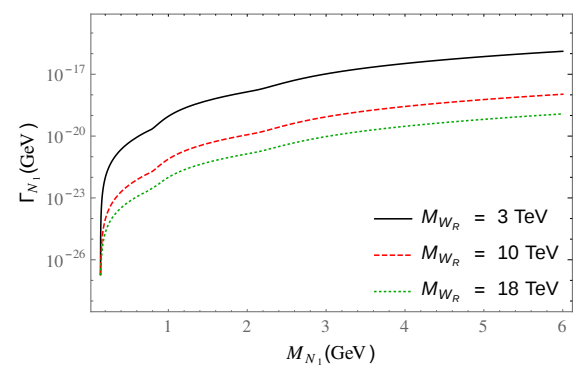

Table 1: Inputs for various experiments.

\begin{tabular}{|c|c|c|c|}
\hline Experiments & $N_{M_{1}}$ & $L_{D}[\mathrm{~m}]$ & $\beta_{M_{1}}[\mathrm{GeV}]$ \\
\hline NA62 & $N_{K}=1.35 \times 10^{13}$ & 170 & 75 \\
\hline Belle II & $N_{D, D_{s}, B}=3.4,1,5.5 \times 10^{10}$ & 1.5 & 0 \\
\hline LHCb & $N_{D, D_{s}, B}=5,2.3,0.8 \times 10^{12}$ & 20 & 100 \\
\hline FCC-ee & $N_{B}=6 \times 10^{11}$ & 2 & $\frac{M_{Z}}{2}$ \\
\hline SHiP & $N_{D, D_{s}}=1.02,0.27 \times 10^{17}$ & 60 & 58 \\
\hline
\end{tabular}

Figure 2: The total decay width of the heavy neutrino $N_{1}$

\section{Limit on $M_{W_{R}}$ from ongoing and future experiments}

The sensitivity reach for the above LNV decay modes in a particular experiment depends on the number of the parent mesons $M_{1}$ 's produced $\left(N_{M_{1}^{+}}\right)$, their momentum $\left(\vec{p}_{M_{1}}\right)$ and the branching ratio for these mesons to the LNV modes. Assuming the parent meson $M_{1}$ decays at rest, the expected number of signal events is [3]:

$$
N_{\text {event }}=2 N_{M_{1}^{+}} \operatorname{Br}\left(M_{1}^{+} \rightarrow \ell^{+} \ell^{+} M_{2}^{-}\right) \mathscr{P}_{N} \approx 2 N_{M_{1}^{+}} \operatorname{Br}\left(M_{1}^{+} \rightarrow \ell^{+} N_{i}\right) \frac{\Gamma\left(N_{i} \rightarrow \ell^{+} M_{2}^{-}\right)}{\Gamma_{N_{i}}} \mathscr{P}_{N},
$$

where for $\ell=e, N_{i}=N_{1}$ and for $\ell=\mu, N_{i}=N_{2}$, the factor 2 is due to inclusion of the charge conjugate process $M_{1}^{+} \rightarrow \ell^{+} N_{i}$ and $\mathscr{P}_{N}$, is the probability of the RH neutrino $N_{i}$ to decay within a detector of the length $L_{D}$ given by: $\mathscr{P}_{N}=\left[1-\exp \left(-\frac{M_{N_{i}} \Gamma_{N_{i}} L_{D}}{p_{N_{i}}^{*}}\right)\right]$. In the above, $p_{N_{i}}^{*}=\frac{m_{M_{1}}}{2} \lambda^{\frac{1}{2}}\left(1, \frac{m_{\ell}^{2}}{m_{M_{1}}^{2}}, \frac{M_{N_{i}}^{2}}{m_{M_{1}}^{2}}\right)$ is the momentum of $N_{i}$ in $M_{1}$ rest frame. For the meson $M_{1}$ produced with fixed boost $\vec{\beta}$, the energy of $N_{i}$ is then given by, $E_{N_{i}}=E_{N_{i}}^{*}\left(\gamma+\frac{p_{N_{i}}^{*}}{E_{N_{i}}^{*}} \sqrt{\gamma^{2}-1} \cos \theta_{N_{i}}^{*}\right)$, where $E_{N_{i}}^{*}, p_{N_{i}}^{*}$ are the energy 
and momentum of $N_{i}$ in rest frame of $M_{1}$ and $\gamma=\frac{E_{M_{1}}}{m_{M_{1}}} . \theta_{N_{i}}^{*}$ is the emission angle of particle $N_{i}$ in the rest frame of $M_{1}$, which is measured from the boost direction $\vec{\beta}$. Hence, the signal event for $M_{1}^{+} \rightarrow \ell^{+} \ell^{+} M_{2}^{-}$in the lab-frame is:

$$
N_{\text {event }} \approx 2 N_{M_{1}^{+}} \int_{E_{N_{i}}^{-}}^{E_{N_{i}}^{+}} d E_{N_{i}} \operatorname{Br}\left(M_{1}^{+} \rightarrow \ell^{+} N_{i}\right) \frac{m_{M_{1}}}{2 p_{N_{i}}^{*}\left|\vec{p}_{M_{1}}\right|} \frac{\Gamma\left(N_{i} \rightarrow \ell^{+} M_{2}^{-}\right)}{\Gamma_{N_{i}}} \mathscr{P}_{N}^{\prime}
$$

where $\mathscr{P}_{N}^{\prime}=\left[1-\exp \left(-\frac{M_{N_{i}} \Gamma_{N_{i}} L_{D}}{\sqrt{E_{N_{i}}^{2}-M_{N_{i}}^{2}}}\right)\right]$, is the probability of $N_{i}$ to decay within the detector length $L_{D}$, after taking into account the boost factor.
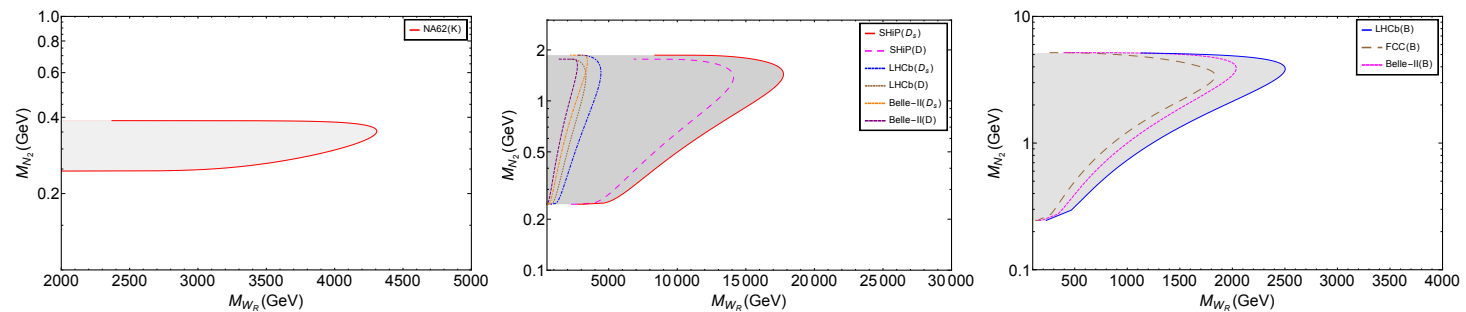

Figure 3: Constraints on the RH gauge boson $M_{W_{R}}$ mass, corresponding to a heavy neutrino $M_{N}$ for $K, D, D_{s}$ and $B$ meson decays.

\section{Input for different Experiments}

In Table.1, we have listed all the relevant input parameter for different experiments which we have used in our calculation.

\section{Results}

From Fig. 3 it is evident that the most stringent limit in the $M_{N} \sim 1 \mathrm{GeV}$ range will be provided by SHiP with the $D_{s} / D \rightarrow \ell^{+} \ell^{+} \pi^{-}$decay mode. In the relatively higher mass range $M_{N} \sim 4 \mathrm{GeV}$, stringent limit can be obtained by FCC-ee, Belle-II and LHCb experiments. Besides, the ongoing NA62 can give constraint $M_{W_{R}}>4.6 \mathrm{TeV}\left(M_{N_{1}} \sim 0.38 \mathrm{GeV}\right)$, which is competitive with the collider bounds from LHC.

\section{Conclusions}

We evaluate the lepton number violating meson decays $M_{1} \rightarrow \ell^{+} \ell^{+} M_{2}$ within the framework of a Left-Right symmetric model. The meson decays are sensitive for low mass right handed neutrinos (in the few $100 \mathrm{MeV}$-few GeV range) and are complementary to LHC (sensitive to few hundred $\mathrm{GeV}$ to $\mathrm{TeV}$ mass neutrinos).

\section{References}

[1] J. C. Pati and A. Salam, Phys. Rev. D 10, 275 (1974).

[2] S. Mandal, M. Mitra and N. Sinha, Phys. Rev. D 96, no. 3, 035023 (2017)

[3] T. Asaka and H. Ishida, Phys. Lett. B 763, 393 (2016). 